\title{
Crescimento de Aegla manuinflata (Decapoda, Anomura, Aeglidae) em ambiente natural
}

\author{
André Trevisan \& Sandro Santos
}

Laboratório de Carcinologia, Universidade Federal de Santa Maria, Av. Roraima 1000, Cidade Universitária, Bairro Camobi, 97105-900 Santa Maria, RS, Brasil. (sandro.santos30@gmail.com)

\begin{abstract}
Growth of Aegla manuinflata (Decapoda, Anomura, Aeglidae) in natural environment. The aim of this work was to evaluate the growth of Aegla manuinflata Bond-Buckup \& Santos, 2009 in natural environment. Samplings were monthly performed, from April/2006 to May/2007, in a tributary of Ibicuí-Mirim River, which belongs to the Uruguay River basin, at São Pedro do Sul municipality, state of Rio Grande do Sul, Brazil. Traps and dip nets were used to collect the animals. The specimens were sexed and had their cephalothoracic length (CL) measured, using a digital caliper with $0.01 \mathrm{~mm}$ precision. The growth was evaluated using the von Bertalanffy model. The growth curves in length (mm), for the data obtained through the modal progression, are described by the equations: $\mathrm{Ct}=28.00\left[1-\mathrm{e}^{-0.0047(t+10.63)}\right]$ for males and $\mathrm{Ct}=25.16[1-$ $\left.\mathrm{e}^{-0.0051(t+17.65)}\right]$ for females. The maximum estimated age for males was three years and for females was two years and six months.
\end{abstract}

KEYWORDS. Age estimative, von Bertalanffy model, recruitment.

RESUMO. O presente trabalho teve como objetivo avaliar o crescimento de Aegla manuinflata Bond-Buckup \& Santos, 2009 em ambiente natural. Para isso foram realizadas coletas mensais de abril de 2006 a maio de 2007 no arroio Passo Taquara, no município de São Pedro do Sul, Rio Grande do Sul, Brasil. Os organismos foram coletados utilizando armadilhas plásticas e puçá. Os espécimes foram sexados e tiveram mensurado o comprimento do cefalotórax (CC), em seguida foram devolvidos ao córrego. O crescimento foi avaliado utilizando o modelo de von Bertalanffy. As curvas de crescimento do cefalotórax, estimadas para machos e fêmeas, são descritas pelas seguintes equações $\mathrm{Ct}=28,00$ [1 $\left.-\mathrm{e}^{-0,0047(t+10,63)}\right]$ e $\mathrm{Ct}=25,16\left[1-\mathrm{e}^{-0,0051(t+17,65)}\right.$, respectivamente. Os machos atingem tamanhos maiores que as fêmeas. A longevidade foi estimada em três anos para os machos e dois anos e meio para as fêmeas.

PALAVRAS-CHAVE. Modelo de von Bertalanffy, estimativa de tempo de vida, recrutamento.

O crescimento em animais pode ser expresso pelo aumento do comprimento, peso, volume, largura, entre outras variáveis, ao longo do tempo (HARTNOLL, 1982). Esta variável é resultante de um balanço entre os processos de anabolismo e catabolismo que ocorrem no indivíduo, sendo que esses dependem de fatores externos como a temperatura e a disponibilidade de alimento, sendo estes especialmente importantes para crustáceos (HARTNOLL, 2001).

De acordo com Hartnoll (1982), os decápodos crescem assintoticamente, com a taxa de crescimento somático relacionada a sucessivas ecdises durante a ontogenia, podendo diferir de acordo com o sexo. As estratégias de crescimento em crustáceos são crescimento indeterminado e crescimento determinado. $\mathrm{Na}$ primeira, os animais continuam a sofrer mudas indefinidamente, sendo que, conforme o tempo passa, o incremento na muda diminui e o período de intermuda aumenta; nesse modelo as taxas de crescimento irão determinar o tamanho final do animal. Em animais com crescimento determinado, as mudas param de ocorrer quando esses atingem a maturidade sexual, ou seja, nesses animais as mudas puberal e final são as mesmas (HARTNOLL, 1982).

A curva de crescimento pode ser determinada para animais isolados, mas com frequência é estimada como uma média populacional, obtida com base nos dados de toda a população estudada ou de parte dela (MARGALEF, 1977).

Em crustáceos, o crescimento pode ser avaliado com base no cultivo de espécies, acompanhando o incremento de tamanho e/ou peso durante os ciclos de muda ou, ainda, pelo acompanhamento das coortes etárias resultantes da distribuição da frequência dos organismos em função do tempo (FonTeles-FilHo, 1987). O modelo matemático proposto por vON BERTALANFFY (1938) tem mostrado resultados satisfatórios no ajuste aos pontos empíricos, sendo que é amplamente utilizado para expressar a relação do tamanho ou peso corpóreo em função da idade dos animais (MunRo, 1982; PINHEIRO $\&$ TADDEI, 2005).

Existem informações significativas sobre o crescimento de Decapoda de águas continentais, destacando-se os trabalhos com Palaemonidae (VALENTI et al., 1987, 1993, 1994; Souza \& Fontoura, 1995; Он et al., 2002; CARTAXANA, 2003), Trychodactylidae (Pinheiro \& Taddei, 2005) e Parastacidae (Noro \& Buckup, 2009). Dentro da família Aeglidae, o modelo de VON BERTALANFFy (1938) foi utilizado para avaliar o crescimento de Aegla platensis Schmitt, 1942, Aegla leptodactyla Buckup \& Rossi, 1977, Aegla longirostri Bond-Buckup \& Buckup, 1994 e Aegla jarai BondBuckup \& Buckup, 1994 (Bueno et al., 2000; Noro \& Buckup, 2003; Silva-Castiglioni et al., 2006 e Boss JR. et al., 2006, respectivamente).

Devido às informações fundamentais que esses estudos fornecem para a compreensão da biologia de eglídeos como, por exemplo, as taxas de crescimento, longevidade, composição de tamanho, entre outros, o presente estudo tem como objetivo caracterizar o crescimento de Aegla manuinflata Bond-Buckup \& Santos, 2009, em ambiente natural, visando obter maiores informações para subsidiar a preservação do grupo. 


\section{MATERIAL E MÉTODOS}

Os espécimes de A. manuinflata foram coletados mensalmente de maio de 2006 a abril de 2007, no arroio Taquara, pertencente à bacia do rio Ibicuí/Uruguai, localizado no município de São Pedro do Sul, região central do Estado do Rio Grande do Sul. As amostragens foram realizadas com a utilização de puçá e armadilhas confeccionadas com garrafas plásticas, distribuídas ao longo do córrego; as mesmas foram colocadas ao anoitecer e retiradas no início da manhã seguinte.

Os indivíduos foram sexados através da observação dos pleópodos nas fêmeas e a ausência destes nos machos, e/ou através da visualização das aberturas genitais (no coxopodito do $3^{\circ}$ par de pereiópodos nas fêmeas e no coxopodito do $5^{\circ}$ par de pereiópodes nos machos); foi mensurado o comprimento cefalotorácico (CC), da ponta do rostro até a borda posterior da carapaça. Foram considerados juvenis todos os animais menores que a menor fêmea ovígera amostrada em campo (12,34 $\mathrm{mm}$ de $\mathrm{CC})$. Após esses procedimentos, os animais foram devolvidos ao arroio.

$\mathrm{O}$ crescimento em comprimento de $A$. manuinflata foi analisado com base na distribuição de frequências absolutas em intervalos de classe de tamanho de 2 $\mathrm{mm}$ de $\mathrm{CC}$, que corresponde à quarta parte do desvio padrão da média calculada para o total da amostra examinada (MARKUS, 1971). Os organismos com menos de três milímetros de comprimento não apresentavam pleópodos e os poros genitais eram difíceis de serem visualizados, o que impossibilitava uma sexagem segura; neste sentido, foram somados ao conjunto de ambos os sexos na análise da distribuição de frequência em classes de tamanho.

O crescimento em comprimento de machos e fêmeas foi estimado separadamente através do deslocamento das modas obtidas, ao longo de cada mês de amostragem (Macdonald \& Pitcher, 1979; MaCDONALD, 1987). As modas dos histogramas de frequência de comprimento de cefalotórax foram obtidas segundo Spiegel (1979): $\mathrm{MODA}=\mathrm{L}_{1}+\left[\Delta_{1} / \Delta_{1}+\right.$ $\Delta_{2}$ ] X C, onde: $\mathrm{L}_{1}=$ limite real inferior da classe modal (a que contém a moda); $\Delta_{1}=$ excesso da frequência modal sobre a classe imediatamente inferior; $\Delta_{2}=$ excesso da frequência modal sobre a classe imediatamente superior e $\mathrm{C}=$ amplitude do intervalo da classe modal.

Foi utilizado o modelo de crescimento de vON BertalanfFy (1938), que corresponde à seguinte equação matemática: $\mathrm{C}_{\mathrm{t}}=\mathrm{C}_{\mu}\left[1-\mathrm{e}^{-\mathrm{k}(\mathrm{t}+\mathrm{to})}\right]$, onde: $\mathrm{C}_{\mathrm{t}}=$ comprimento médio do cefalotórax dos indivíduos com idade $\mathrm{t}$, em $\mathrm{mm} ; \mathrm{C}=$ comprimento médio máximo da carapaça, em $\mathrm{mm} ; \mathrm{k}^{\mu}=$ parâmetro relacionado com a taxa de crescimento, referente a dias; $\mathrm{e}=$ base dos logaritmos naturais; $\mathrm{t}=$ idade dos indivíduos, em dias; $\mathrm{t}$, parâmetro relacionado com o comprimento do animal ao nascer.

As curvas de crescimento foram linearizadas de acordo com Allen (1976) e as retas obtidas foram comparadas através de uma análise de covariância, para um intervalo de confiança de 95\% (SNEDECOR \& Cochran, 1967). Nesse teste foram avaliadas três hipóteses: $1^{a}$ hipótese da homogeneidade das variâncias residuais; $2^{\mathrm{a}}$ hipótese da igualdade entre a declividade e elevação das retas de machos e fêmeas e $3^{\mathrm{a}}$ hipótese da igualdade entre as intersecções das retas de machos e fêmeas.

\section{RESULTADOS E DISCUSSÃO}

Foram amostrados 888 indivíduos, sendo 17 juvenis não sexados, 572 machos e 299 fêmeas. O menor indivíduo capturado foi um juvenil não sexado com 1,76 $\mathrm{mm}$ de $\mathrm{CC}$ e o maior um macho adulto com $27,9 \mathrm{~mm}$ de CC.

A maior frequência de juvenis foi observada na primavera, principalmente nos meses de outubro e novembro, como pode ser observado nas distribuições de frequências absolutas do comprimento cefalotorácico para ambos os sexos (Figs. 1, 2). Por esse motivo, optouse por acompanhar o crescimento dos animais a partir dessa época do ano e decidiu-se incorporar ao estudo do deslocamento modal a coorte de juvenis amostrados a partir desses meses, antepondo-se ao grupo dos animais maiores, amostrados no período anterior. Esse procedimento foi adotado também por NoRO \& Buckup (2003) e Silva-CASTiglioni et al. (2006), que mencionaram que esse procedimento é adequado em virtude do crescimento em populações locais não sofrer grandes alterações de um ano para o outro.

Com base na presença de indivíduos de menor tamanho durante o ano todo é possível concluir que esta população exibe um recrutamento contínuo, porém, com maior intensidade na primavera e no inverno; este fato é corroborado pela presença de fêmeas ovígeras em todas as estações do ano. Na maioria dos estudos sobre as diferentes espécies de eglídeos tem se sugerido um pulso único de recrutamento ao longo do ano; é o caso do observados em Aegla laevis laevis Latreille, 1818 (BAHAMONDE \& LoPEZ, 1961), Aegla paulensis Schmitt, 1942 (Lopez, 1965), Aegla perobae Hebling \& Rodrigues, 1977 (Rodrigues \& Hebling, 1978), A. platensis (BuEno et al., 2000), A. leptodactyla (Noro \& Buckup, 2003), A. jarai (Boss Jr. et al., 2006), A. longirostri (Silva-Castiglioni et al., 2006) e $A$. itacolomiensis (Silva-Gonçalves et al., 2009). No entanto um recrutamento mais prolongado pode ocorrer como relata Swiech-Ayoub \& Masunari (2001a) em seus estudos sobre a biologia reprodutiva de $A$. castro Schmitt, 1942.

As curvas de crescimento em comprimento cefalotorácico, de machos e fêmeas, correspondem às seguintes equações, respectivamente: $\mathrm{Ct}=28,00$ [1 $\left.-\mathrm{e}^{-0,0047(\mathrm{t}+10,63)}\right](\mathrm{n}=601 \mathrm{r}=0,94)$ e $\mathrm{Ct}=25,16[1-\mathrm{e}$ $-0,0051(t+17,65)](n=298 ; r=0,96)$ (Figs. 3, 4).

$\mathrm{O}$ valor médio máximo do $\mathrm{CC}$, estimado para os 

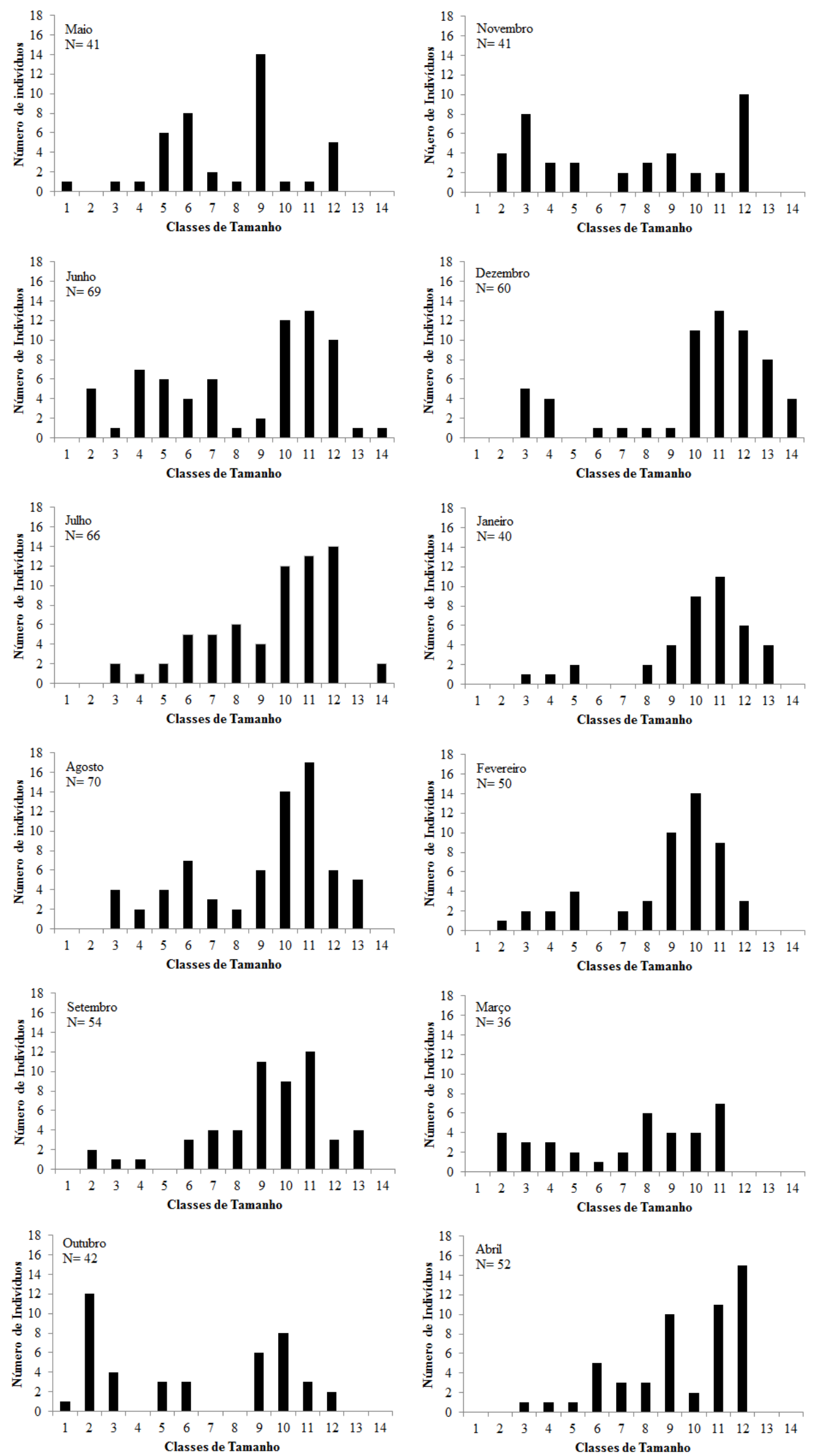

Fig. 1. Distribuição da frequência absoluta de machos de Aegla manuinflata Bond-Buckup \& Santos, 2009 nas classes de comprimento do


e (14) $26 \nmid 28$ 

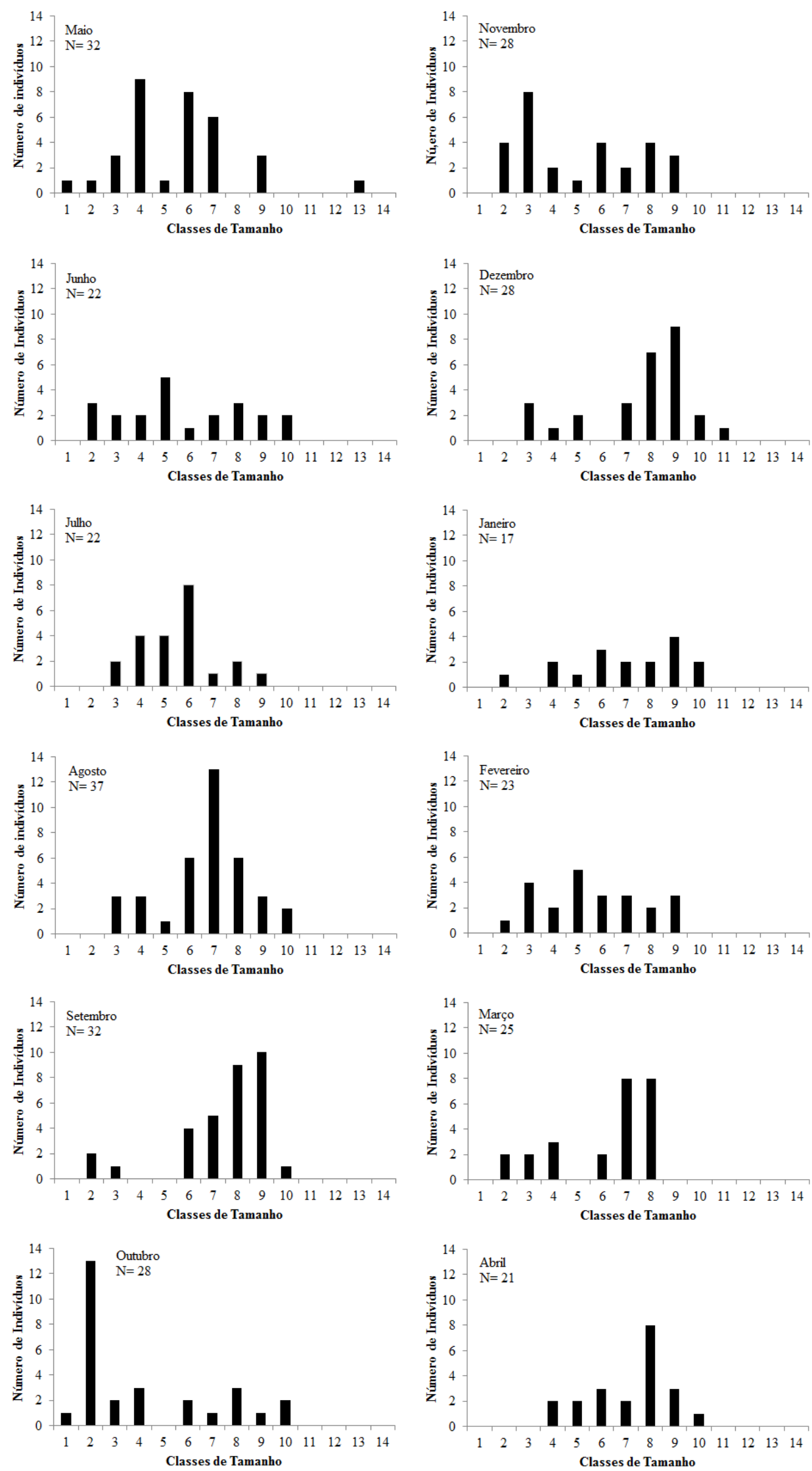

Fig. 2. Distribuição da frequência absoluta de fêmeas de Aegla manuinflata Bond-Buckup \& Santos, 2009 nas classes de comprimento do


e (14) $26+28$. 


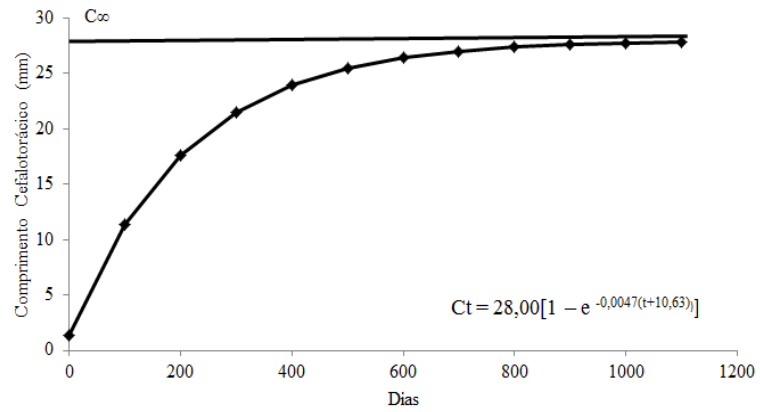

Fig. 3. Curva de crescimento de machos de Aegla manuinflata BondBuckup \& Santos, 2009 (Ct, comprimento do cefalotórax no tempo t; $\mathrm{C} \alpha$, comprimento médio máximo do cefalotórax, em mm; t, idade em dias).

machos através do deslocamento modal, foi de 28,00 $\mathrm{mm}$; para as fêmeas esse valor foi um pouco menor, 25,13 $\mathrm{mm}$. Esses valores são próximos daqueles observados no maior macho $(27,9 \mathrm{~mm}$ de $\mathrm{CC})$ e na maior fêmea $(24,92 \mathrm{~mm}$ de $\mathrm{CC})$ coletados na natureza.

Os valores do crescimento assintótico não foram semelhantes entre os sexos de $A$. manuinflata, sendo esta uma característica do grupo já observada nos trabalhos de crescimento realizados com A. leptodactyla, $A$. longirostri, A. jarai e A. itacolomiensis (Noro \& BUCKuP, 2003; Silva-CASTiglioni et al., 2006; Boss JR. et al., 2006 e Silva-Gonçalves et al., 2009, respectivamente). Além destes, o tamanho diferencial entre os sexos é registrado em A. laevis laevis (BAhamonde \& Lopez, 1961), A. paulensis (Lopez, 1965), A. perobae (Rodrigues \& Hebling, 1978), A. platensis (Bueno \& Bond-Buckup, 2000), A. castro (Swiech-Ayoub \& Masunari, 2001b), $A$. franciscana (GonçALves et al., 2006), Aegla uruguayana Schmitt, 1942 (VIAU et al., 2006), Aegla franca Schmitt, 1942 (Bueno \& Shimizu, 2009), entre outros.

Swiech-Ayoub \& Masunari (2001b) relataram que geralmente machos alcançam tamanhos maiores que as fêmeas por direcionarem suas energias principalmente para o crescimento somático, enquanto nas fềmeas grande parte da energia é gasta no processo reprodutivo (maturação de gônadas, desenvolvimento dos ovos, cuidado parental, entre outros), em detrimento do crescimento do corpo.

A taxa de crescimento de $A$. manuinflata foi semelhante em machos $(\mathrm{k}=0,0047)$ e fêmeas $(\mathrm{k}=0,0051)$, como observado em A. platensis e A. leptodactyla (Bueno et al., 2000; Noro \& Buckup, 2003). Em A. manuinflata foram observados valores de $\mathrm{k}$ semelhantes entre machos e fêmeas (levemente maior no segundo grupo), como observado também em $A$. leptodactyla (Noro \& Buckup, 2003); este fato pode estar associado a alguns fatores que podem alterar a composição do tamanho de machos e fêmeas desses animais, em ambiente natural, como a mortalidade diferencial entre os sexos, migrações diferenciais, capacidade de suportar adversidades ambientais, desequilíbrio espaço-temporal na utilização dos recursos alimentares e padrões comportamentais diferenciados entre os sexos (GIESEL, 1972; Wolf et al., 1975; Montague, 1980).

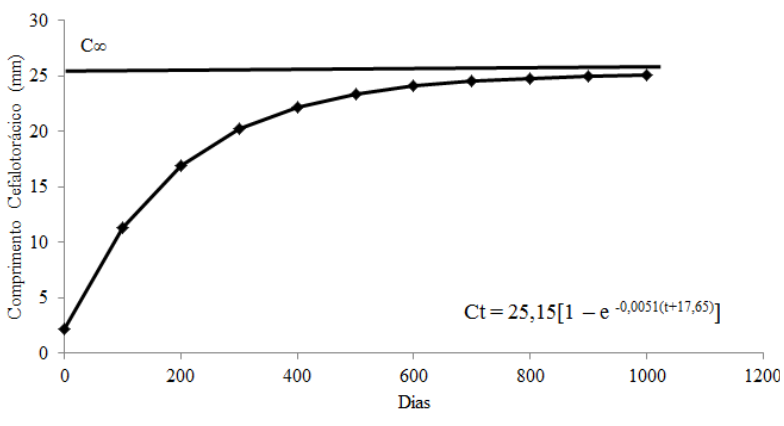

Fig. 4. Curva de crescimento de fêmeas de Aegla manuinflata BondBuckup \& Santos, 2009 (Ct, comprimento do cefalotórax no tempo t; $\mathrm{C} \alpha$, comprimento médio máximo do cefalotórax, em $\mathrm{mm}$; t, idade em dias).

Por outro lado, em A. longirostri (SILVACAstiglioni et al., 2006), A. jarai (Boss JR. et al., 2006) e A. itacolomiensis (Silva-Gonçalves et al., 2009), foram observados valores de $\mathrm{k}$ maiores nos machos. Conforme Hartnoll $(1982,1985)$ machos e fêmeas crescem de maneira semelhante até a maturidade sexual; após esse período as fêmeas crescem menos devido a longos períodos de intermuda associadas principalmente à postura dos ovos. Além disso, o crescimento e a reprodução são eventos antagônicos em crustáceos, ou seja, a energia disponibilizada para a reprodução não é disponibilizada para o crescimento somático; esses fatores podem explicar os valores mais elevados de $\mathrm{k}$ para os machos daquelas espécies.

$\mathrm{O}$ tamanho maior em machos de A. manuinflata, mesmo estes apresentando taxas de crescimento menores, provavelmente está associado a sua maior longevidade (3 anos para os machos contra 2,5 anos para as fêmeas), uma vez que, vivendo mais tempo, eles devem passar por mais mudas, alcançado tamanhos maiores que as fêmeas. Vale ressaltar que esta espécie, assim como outros eglídeos, apresentam crescimento indeterminado, o que pode ser evidenciado pela presença de fêmeas ovígeras com diferentes tamanhos.

O modelo de crescimento aplicado neste trabalho permitiu estimar uma longevidade de aproximadamente três anos para A. manuinflata. Nos demais trabalhos onde a longevidade de eglídeos também foi calculada pelo modelo vON BeRTALANFFy (1938), a estimativa deste parâmetro populacional apresenta variações sutis. A longevidade de A. laevis laevis foi estimada em aproximadamente 37 meses (BAHAMONDE \& Lopez, 1961) e de 2,5 anos em A. platensis (BuENo et al., 2000). Valores de longevidade um pouco menor são registrados para A. jarai (Boss JR. et al., 2006) e A. longirostri (Silva-Castiglioni et al., 2006). No trabalho com $A$. leptodactyla (Noro \& Buckup, 2003) a longevidade estimada foi de 2,5 anos, porém a longevidade ficou subestimada no estudo da curva de crescimento, assim sendo os autores observaram que aos dois anos e meio os machos de A. leptodactyla poderiam atingir $17,69 \mathrm{~mm}$ de comprimento cefalotorácico. SilvaGonçaLves et al. (2009) registraram que a logenvidade 
Tab. I. Comparação entre os componentes da equação de crescimento de von Bertalanffy e longevidade em Aegla (C $\alpha$, comprimento médio máximo da carapaça; k, taxa de crescimento; to, tempo de vida, em dias, do menor espécime da amostra).

\begin{tabular}{|c|c|c|c|c|c|c|c|c|}
\hline Espécie & Referência & $\mathrm{C} \alpha \widehat{\jmath}$ & $\mathrm{C} \alpha+$ & $\mathrm{k} \sigma^{\lambda}$ & $\mathrm{k}$ 우 & to $\hat{\sigma}$ & to우 & $\begin{array}{l}\text { Longevidade } \\
\text { (anos) }\end{array}$ \\
\hline A. manuinflata & Presente estudo & 28,00 & 25,16 & 0,0047 & 0,0051 & 10,63 & 17,65 & 3,0 \\
\hline A. platensis & Bueno et al. (2000) & 17,39 & 19,12 & 0,0041 & 0,0033 & 39,13 & 50,38 & 2,5 \\
\hline A. leptodactyla & Noro \& Buckup (2003) & 19,83 & 18,96 & 0,0023 & 0,0024 & 55,5 & 48,6 & 2,5 \\
\hline A. jarai & Boss JR. et al. (2006) & 25,11 & 23,56 & 0,0082 & 0,0048 & 29,02 & 46,45 & 2,0 \\
\hline A. longirostri & CASTiglioni et al. (2006) & 27,9 & 21,45 & 0,0069 & 0,0048 & 24,78 & 45,62 & 2,0 \\
\hline A. itacolomiensis & SiLVA-GonÇALVEs et al. (2009) & 23,21 & 19,49 & 0,0094 & 0.0065 & 7,03 & 11,16 & 2,5 \\
\hline
\end{tabular}

de A. itacolomiensis foi de 2,2 e 2,5 anos para machos e fêmeas, respectivamente.

No presente trabalho, as fêmeas apresentaram uma longevidade menor que a dos machos, fato semelhante ao observado em A. laevis laevis (BAHAMONDE \& Lopez, 1961). A maior longevidade dos machos de $A$. manuinflata pode estar relacionada a uma maior taxa de mortalidade de fêmeas maduras causada pela predação, pois a massa de ovos que as mesmas carregam junto ao abdome durante a incubação pode torná-las menos ágeis para a fuga; outro fator que pode aumentar a mortalidade de fêmeas é o estresse sofrido em virtude da postura de ovos, fato que as deixaria mais vulneráveis (SWIECHAyoub \& Masunari, 2001b).

Silva-Castiglioni et al. (2006) e Silva-Gonçalves et al. (2009) verificaram longevidade um pouco maior nas fêmeas de $A$. longirostri e $A$. itacolomiensis, respectivamente. Os autores atribuem esse fato ao comportamento dos machos, que permanecem mais tempo expostos na superfície alimentando-se, disputando e cortejando fêmeas e, assim, ficariam mais expostos à predação e adversidades ambientais; da mesma forma, é discutido que espécies que vivem em ambientes com condições mais semelhantes de fotoperíodo e temperaturas semelhantes tendem a crescer de modo mais semelhante.

Uma comparação entre os valores do comprimento médio máximo da carapaça $(\mathrm{C} \alpha)$, taxa de crescimento (k), tempo de vida em dias do menor animal da amostra (to) e longevidade das espécies de Aegla que tiveram seu crescimento avaliado pelo modelo de VON BERTALANFFY (1938) e o presente trabalho pode ser observados na Tabela I.

$\mathrm{Na}$ comparação das curvas de crescimento linearizadas de $A$. manuinflata foi observado que as mesmas não apresentam diferenças quanto à homogeneidade das variâncias residuais $(\mathrm{Fc}=0,31$; $\mathrm{p}>0,05)$. Da mesma forma não foram observadas diferenças na inclinação entre as retas de machos e fêmeas $(\mathrm{Fb}=0,58 ; \mathrm{p}>0,05)$, porém as retas apresentaram elevações diferenciadas $(\mathrm{Fa}=46,27 ; \mathrm{p}<0,05)$, o que reforça a constatação de um crescimento diferenciado entre machos e fêmeas de $A$. manuinflata.

Os dados obtidos na aplicação do modelo de crescimento proposto por VON BERTALANFFY (1938) se mostraram adequados ao estudo do crescimento de $A$. manuinflata em ambiente natural, fornecendo informações valiosas sobre a taxa de crescimento, crescimento diferencial entre os sexos, longevidade e comprimento médio máximo alcançado por machos e fêmeas. Estudos que descrevem tais parâmetros populacionais podem servir de base para a criação de medidas de conservação dos ambientes de água doce, contribuindo para a conservação da biodiversidade desses locais.

Agradecimentos. Os autores agradecem ao Programa de Pós-Graduação em Biodiversidade Animal e à equipe do Laboratório de Carcinologia da Universidade Federal de Santa Maria, pela ajuda durante a elaboração deste trabalho. A Capes pela bolsa de mestrado a $\mathrm{AT}$ e ao CNPq pela bolsa de pesquisa a SS.

\section{REFERÊNCIAS BIBLIOGRÁFICAS}

Allen, R. L. 1976. Method for comparing fish growth curves. New Zealand Journal of Marine and Freshwater Research 10(Supl. 4):687-692.

BAHAMONDE, N. \& Lopez, M. T. 1961. Estudios biologicos en la populacion de Aegla laevis laevis (Latreille) de el Monte (Crustacea, Decapoda, Anomura). Investigaciones Zoológicas Chilenas 7:19-58.

BertalanfFy, L. vON. 1938. A quantitative theory of organic growth. Human Biology 10(2):181-213.

Boss JR., H.; Silva-Castiglioni, D.; Schacht, K.; Buckup, L. \& BondBuckup, G. 2006. O crescimento de Aegla jarai Bond-Buckup \& Buckup (Crustacea, Anomura, Aeglidae). Revista Brasileira de Zoologia 23(2):490-496.

Bueno, A. A. P. \& Bond-Buckup, G. 2000. Dinâmica populacional de Aegla platensis Schmitt (Crustacea, Decapoda, Aeglidae). Revista Brasileira de Zoologia17(1):43-49.

Bueno, A. A. P.; Bond-Buckup, G. \& Buckup, L. 2000. Crescimento de Aegla platensis em ambiente natural (Crustacea, Decapoda, Aeglidae). Revista Brasileira de Zoologia17(1):51-60.

Bueno, S. L. DE S. \& Shimizu, R. M. 2009. Allometric growth, sexual maturity, and adult male chelae dimorphism in Aegla franca (Decapoda: Anomura: Aeglidae). Journal of Crustacean Biology 29(3):317-328.

Cartaxana, A. 2003. Growth of the prawn Palaemon longirostris (Decapoda, Palaemonidae) in Mira River and estuary, SW Portugal. Journal of Crustacean Biology 23(2):251-257.

Fonteles-FilHo, A. A. 1987. Recursos pesqueiros, biologia e dinâmica populacional. Fortaleza, Imprensa Oficial do Ceará. 296 p.

GieseL, J. T. 1972. Sex ratio, rate of evolution, and environmental heterogeneity. American Naturalist 106:380-387.

Gonçalves, R. S.; Castiglioni, D. S. \& Bond-Buckup, G. 2006. Ecologia populacional de Aegla franciscana (Crustacea, Decapoda, Anomura) em São Francisco de Paula, RS, Brasil. Iheringia, Série Zoologia 96(1):109-114.

Hartnoll, R. G. 1982. Growth. In: Bliss, D. E. ed. The Biology of Crustacea, Embriology, Morphology and Genetics. New York, Academic Press. v. 2, p. 111-196. 
1985. Growth, sexual maturity and reproductive output, In: Wenner, A. M. ed. Crustacean Issues: Factors in Adult Growth. Rotterdam, A. A. Balkema. p. 101-128.

2001. Growth in Crustacea: twenty years on. Hydrobiologia 449(1-3): 111-122

Lopez, M. T. 1965. Estudios biologicos en Aegla odebrechtii paulensis Schmitt (Crustacea, Decapoda, Anomura). Boletim de Zoologia da Faculdade de Filosofia, Ciências e Letras da USP 25:301315 .

MargaleF, R. 1977. Ecologia. Barcelona, Ediciones Omega, 951p.

MARKUS, R. 1971. Elementos de estatística aplicada. Porto Alegre, Faculdade de Agronomia e Veterinária da UFRGS, Centro Acadêmico Leopoldo Cortez. 329p.

Macdonald, P. D. M. 1987. The analysis of length-frequency distributions. In: Summerfelt, R. C. \& Hall, G. eds. Age and growth of fish. Ames, Iowa State University Press. p. 371-384.

Macdonald, P. D. M. \& Pitcher, J. 1979. Age-groups from sizefrequency data: a versalite and efficient method of analyzing distribution mixtures. Journal of the Fisheries Research Board of Canada 36:987-1001.

Montague, C. L. 1980. A natural history of temperate Western Atlantic fiddler crabs (Genus $U c a$ ) with reference to their impact on the salt marsh. Contributions of Marine Science 23:25-55.

MunRo, J. L. 1982. Estimation of the parameters of the Von Bertalanffy growth equation from recapture data at variable time intervals. Conseil International pour l'Exploration de la Mer 40:199200.

Noro, C. K. \& Buckup, L. 2003. O crescimento de Aegla leptodactyla Buckup \& Rossi (Crustacea, Anomura, Aeglidae). Revista Brasileira de Zoologia 20(2):191-198.

2009. O crescimento de Parastacus defossus (Crustacea: Decapoda: Parastacidae). Zoologia 26(1):54-60.

Оh, C. W.; Suh, H. L.; Park, K. Y.; Ma, C. W. \& Lim, H. S. 2002. Growth and reproductive biology of the freshwater shrimp Exopalaemon modestus (Decapoda: Palaemonidae) in a lake of Korea. Journal of Crustacean Biology 22(2):357-366.

Pinheiro, M. A. A. \& Taddei, F. G. 2005 Crescimento do caranguejo de água doce, Dilocarcinus pagei Stimpson (Crustacea, Brachyura, Trichodactylidae). Revista Brasileira de Zoologia 22(3):522-528.

Rodrigues, W. \& Hebling, N. J. 1978. Estudos biológicos em Aegla perobae Hebling \& Rodrigues, 1977 (Decapoda, Anomura). Revista Brasileira de Biologia 38(2):383-390.
Silva-Castiglioni, D.; Barcelos, D. F. \& Santos, S. 2006. O crescimento de Aegla longirostri Bond-Buckup \& Buckup (Crustacea, Anomura, Aeglidae). Revista Brasileira de Zoologia 23(2):408-413.

Silva-Gonçalves, R.; Bond-Buckup, G. \& Buckup, L. 2009.Crescimento de Aegla itacolomiensis (Crustacea, Decapoda) em um arroio da Mata Atlântica no sul do Brasil. Iheringia, Série Zoologia 99(4):397-402.

Snedercor, G. W. \& Cochran, W. G. 1967. Statistical Methods. 6ed. Ames, Iowa State University Press. 593p.

SouzA, G. D. \& Fontoura, N. F. 1995. Crescimento de Macrobrachium potiuna no Arroio Sapucaia, município de Gravataí (Crustacea, Decapoda, Palaemonidae). Revista Brasileira de Biologia 55(1):51-63.

Spiegel, M. R. 1979. Estatística. McGraw-Hill do Brasil, São Paulo. 580 p.

Swiech-Ayoub, B. P. \& Masunari, S. 2001a. Biologia reprodutiva de Aegla castro Schmitt (Crustacea, Anomura, Aeglidae) no Buraco do Padre, Ponta Grossa, Paraná, Brasil. Revista Brasileira de Zoologia18(3): 1019-1030.

2001b. Flutuações temporal e espacial de abundância e composição de tamanho de Aegla castro Schmitt (Crustacea, Anomura, Aeglidae) no Buraco do Padre, Ponta Grossa, Paraná, Brasil. Revista Brasileira de Zoologia18(3):1003-1017.

Valenti, W. C.; Mello, J. T. C. \& Castagnolli, N. 1993. Efeito da densidade populacional sobre as curvas de crescimento de Macrobrachium rosenbergii (de Man) em cultivo semi-intensivo (Crustacea, Palaemonidae). Revista Brasileira de Zoologia 10(3):371-558.

Valenti, W. C.; Mello, J. T. C. \& Lobão, V. L. 1987. Crescimento de Macrobrachium acanthurus (Wiegman, 1863) do Rio Ribeira de Iguape (Crustacea, Decapoda, Palaemonidae). Revista Brasileira de Biologia 47(3):349-355.

1994. Maturation and growth curves of Macrobrachium carcinus (Linnaeus) (Crustacea, Decapoda, Palaemonidae) from Ribeira de Iguape River, southern Brazil. Revista Brasileira de Zoologia 11(4):649-658.

Viau, V. E.; López Greco, L. S.; Bond-Buckup, G. \& Rodríguez, E. M. 2006. Size at onset of sexual maturity in anomuran crab, Aegla uruguayana (Aeglidae). Acta Zoologica 87:253-264.

Wolf, P.; Shanholtaer, S. F. \& Reimold, R. J. 1975. Population estimates for Uca pugnax on Duplin estuary marsh, Georgia, USA. Crustaceana 29:79-91.

Recebido em 12 de maio de 2011. Aceito em 30 de novembro de 2011. ISSN 0073-4721

Artigo disponível em: www.scielo.br/isz

Impresso e distribuído em 2012. 